\title{
Multidisciplinary Treatment for Critical Limb Ischemia in Peripheral Arterial Disease
}

\author{
Hiroyoshi Komai, MD, PhD
}

Critical limb ischemia (CLI) is a severe blockage of the arteries to the lower limbs characterized by poor prognoses for both salvage of the lower limb and patient survival. Accordingly, CLI must be diagnosed and treated appropriately from the earliest possible stage. To do so, multidisciplinary treatment not only by vascular surgeons but also by many other doctors and medical staff is necessary. Accurate diagnosis is indispensable to appropriate treatment of CLI; thus, the definitions in the recently issued new guidelines for CLI treatment are reviewed. The multidisciplinary treatment of CLI should be recognized as three elements: namely, multidisciplinary treatment to salvage the lower limb, to improve of survival prognosis, and to prevent CLI occurrence. In all of these events, team medicine administered by expert staff is indispensable. The specialist must have not only profound knowledge of his/her field of specialty but also professional skills and the ability to cooperate with other departments. A multidisciplinary treatment approach that combines the abilities of many specialists for treating severely ischemic limbs in patients with peripheral arterial disease is expected to improve both limb salvage and patient survival and should be promoted in daily clinical settings. (This is a translation of Jpn J Vasc Surg 2018; 27: 507-512.)

Keywords: critical limb ischemia, multidisciplinary treatment, guideline, team medicine, early detection

\section{Introduction}

Peripheral arterial disease $(\mathrm{PAD})$ is a critical condition

Department of Vascular Surgery, Kansai Medical University Medical Center, Moriguchi, Osaka, Japan

Received: February 21, 2019; Accepted: February 21, 2019 Corresponding author: Hiroyoshi Komai, MD, PhD. Department of Vascular Surgery, Kansai Medical University Medical Center, 10-15 Fumizonocho, Moriguchi, Osaka 570-8507, Japan

Tel: +81-6-6992-1001, Fax: +81-6-6992-9477

E-mail: komaihir@takii.kmu.ac.jp

This is a translation of Jpn J Vasc Surg 2018; 27: 507-512.

(cc) BY-NC-SA (C2019 The Editorial Committee of Annals of Vascular Diseases. This article is distributed under the terms of the Creative Commons Attribution License, which permits use, distribution, and reproduction in any medium, provided the credit of the original work, a link to the license, and indication of any change are properly given, and the original work is not used for commercial purposes. Remixed or transformed contributions must be distributed under the same license as the original. that may result in loss of limbs or worse life prognosis as it increases in severity. Critical limb ischemia (CLI) must therefore be accurately diagnosed at an early stage and treated appropriately. To date, although PAD has been handled by vascular surgeons, with the disease pathology being better understood in recent times, it is being considered as a disease that can never be dealt with by a single department or a single type of medical professional. In recent years, multidisciplinary treatment has attracted attention with respect to several diseases, but it is now becoming important in the treatment of PAD. In this paper, I have described multidisciplinary treatment for CLI patients, who are with the poorest prognosis in PAD, based on my current understanding.

\section{What is Multidisciplinary Treatment?}

Multidisciplinary treatment is a term that is often used in the treatment of malignant neoplasms, and it represents comprehensive treatment that combines therapies from various disciplines of surgery, internal medicine, and radiation. Multidisciplinary treatment of PAD/CLI is often defined as the combination of bypass surgery, endovascular treatment, wound healing, or rehabilitation therapy that is performed for the purpose of saving limbs and lives. In other words, such intensive and complementary therapies performed from various perspectives using multiple methods are essential for successfully treating CLI. Such a multidisciplinary treatment approach should be applied primarily for the benefit of patients, irrespective of physicians' favor or specialty. Thus, the treatment should always be administered, attempting their best to understand the feelings of patients and their families. The team approach to healthcare which has recently gained attention is a type of multidisciplinary treatment. This is a beneficial form of patient care that can be provided from multiple perspectives, which covers any aspect that may have been overlooked by a single physician or clinical staff. There are several aspects to multidisciplinary treatment for CLI. I have first discussed the definition and diagnosis of severe foot lesions that have been changing over the past few years. I have next described the multidisciplinary treatment for CLI in three elements with the approaches 
adopted in our hospital, including: (1) multidisciplinary treatment for foot lesions, (2) multidisciplinary treatment for improving prognosis, and (3) multidisciplinary treatment for preventing increase in severity.

\section{Definition and Diagnosis of Severe Foot Lesions}

Accurate diagnosis will be required before initiating the treatment of severe foot lesions. The definition of CLI is given by The Trans-Atlantic Inter-Society Consensus Document on Management of Peripheral Arterial Disease (TASC) international guideline published in 2000 and by TASC II, ${ }^{1}$ which is the 2007 revised edition of the former. These guidelines form the basis of the Japanese 2009 PAD guideline and the 2015 revised edition ${ }^{2}$ created by societies centered on The Japanese Circulation Society.

(1) Ischemic symptoms that are present for $>2$ weeks; (2) possibility of other disorders (such as acute arterial occlusion, diabetic gangrene, and connective tissue disorders) has been denied; (3) severe ischemia is proved by objective parameters (such as pain at rest: ankle blood pressure $<50 \mathrm{mmHg}$ or toe blood pressure $<30 \mathrm{mmHg}$ and ulcer or necrosis: ankle blood pressure $<70 \mathrm{mmHg}$ or toe blood pressure $<50 \mathrm{mmHg}$ ); (4) ideally, ischemia proved by transcutaneous oxygen measurements $\left(\mathrm{TcPO}_{2}\right.$; $\mathrm{TcPO}_{2}<30 \mathrm{mmHg}$ ); skin perfusion pressure (SPP) is often used instead of $\mathrm{TcPO}_{2}$ in Japan, such that CLI is diagnosed based on $40 \mathrm{mmHg}$ SPP that is considered equivalent to approximately $30 \mathrm{mmHg} \mathrm{TcPO}_{2}$.

However, it is now being perceived that the treatment of severe peripheral lesions is insufficient when considered based on this concept alone. It has been understood that necrosis of the lower limbs will advance quickly unless appropriate treatment for CLI is provided early. However, there are some cases of CLI that exist but do not advance despite not receiving any treatment. ${ }^{3)}$ Conversely, some wounds end up being subjected to lower limb amputation even when the degree of ischemia is mild. Thus, the appropriateness of making decisions on therapeutic strategies that are solely based on the degree of ischemia is now being questioned. New definitions of severe lower limb lesions have been announced by the United States and Europe. In 2014, the Society of Vascular Surgery proposed the principle called the WIfI classification. ${ }^{4)}$ This is a method for evaluating the prognosis of lower limb by considering the following three factors: wound (W), ischemia (I), and foot infection (fI). The combination of these three factors is expected to allow classification in stages by predicting the prognosis of the lower limb, which is equivalent to the classification system of malignant neoplasms. Compared with the conventional method, the prognosis of the lower limb can be determined more accurately by con-

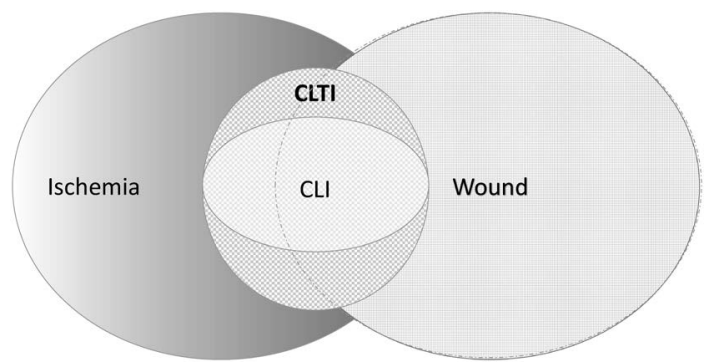

Fig. 1 Definition of chronic limb threatening ischemia (CLTI).

sidering the size of the wound, location of the wound, or presence/absence of complicating infections. Accordingly, a more precise therapeutic strategy can be developed.

Similarly, the PAD guidelines by the European Society for Cardiology (ESC) $)^{5)}$ were revised in 2017. In this edition, CLI was replaced by the new concept of chronic limb threatening ischemia (CLTI). Ischemia based on chronic arterial occlusive disease forms the basis of this CLTI, but it is defined as a case that actually requires treatment for saving the lower limbs, which in turn is determined by considering the degrees of infections and wounds on top of the degree of ischemia. The aforementioned WIfI staging will be considered during decision making. I expect this concept to, from now onward, serve as the basis of development of therapeutic strategies for severe lower limb lesions. Apart from the subject from multidisciplinary treatment, another major characteristic of the revision of this guideline was the change in the first line treatment of below-the-knee lesions from endovascular treatment to the great saphenous vein bypass surgery. Moreover, the levels of recommendation was highly evaluated. The recommendation in the new guideline that was prepared by the collaboration of professionals other than surgeons reflects the fact that expectations toward surgeons is high and the need for distal bypass was re-acknowledged.

The Global Vascular Guideline that is to be published in the near future is expected to become the international guideline substituting the current TASC, and it will adopt the definition of CLTI that is almost equivalent to that given in the ESC guideline. CLTI is expected to become a common term in this field, as in Japan, and therefore, it is necessary that its definition is well understood by physicians in the related fields and by medical staff for making appropriate discussions (Fig. 1).

\section{Multidisciplinary Treatment for Foot Lesions}

Multidisciplinary treatment is currently already performed across several institutions for the treatment of the feet. Endovascular treatment and bypass surgery must be performed without mistaking the indication for the treatment of ischemia. Indeed, safe and precise treatment may 
be provided by combining these therapies (also known as hybrid therapy). The primary therapy for revascularization varies among institutions, and the departments that play the central role may vary among vascular surgery, cardiology, or radiology. The strengths and limitations of each department must be well discussed, and therapeutic strategies developed must be changed until they become patient orientated. Toward this end, it is desirable for each department to be able to casually discuss cases with each other and to develop a strategy together through effective discussion. This is why multidisciplinary treatment is a type of team approach strategy to healthcare. Misjudgment in the method of revascularization or in therapeutic strategies may result in undesirable outcomes for patients; however, it is believed that most of these strategies may have been selected with the best intentions. ${ }^{6}{ }^{6}$ To avoid this, treatment that can support patients up to recovery is required. This is also the case for wound treatment and care. Treatment for wounds may not be provided sufficiently in vascular surgery, and appropriate therapies such as the use of topical agents, wound protection equipment, debridement, vacuum assisted closure (VAC), maggot therapy, or skin grafting must be performed by collaborative efforts of departments such as dermatology and plastic surgery. As a first step toward this initiative, the diagnosis of whether the wound is solely caused by ischemia must be performed by the joint efforts of physicians including dermatologists, diabetologists, and rheumatologists with the support of medical staffs such as vascular medical technicians (CVT), radiological technologists, and clinical engineers. Revascularization is not always sufficient, thus the patient requires adjunctive therapy, for example, vasodilator therapy, low-density lipoprotein (LDL) apheresis, therapeutic angiogenesis, or nerve block. These interventions may also be performed by physicians who belong to the applicable departments. Foot care must be continued after completion of revascularization and wound healing; hence, apart from physicians, various medical staffs must contribute with their expertise to provide patientorientated treatment. Such medical staffs include nurses such as wound, ostomy and continence (WOC) nurses or certified nurse in diabetes nursing, ${ }^{7)}$ physiotherapists, health fitness programmer, biomedical equipment technician, prosthetist, and orthotist, among others. Figure 2 presents the course of therapy of a 71-year-old patient's foot, who visited our hospital after being recommended to undergo right lower limb amputation in another hospital's cardiovascular surgery department. Lower limb artery bypass surgery and Lisfranc amputation were accordingly performed, but wound dehiscence occurred, leading to exposure of the bone. Fortunately, the infection could be controlled and the wound healed after several months of dermatological interventions, including VAC. Since then,

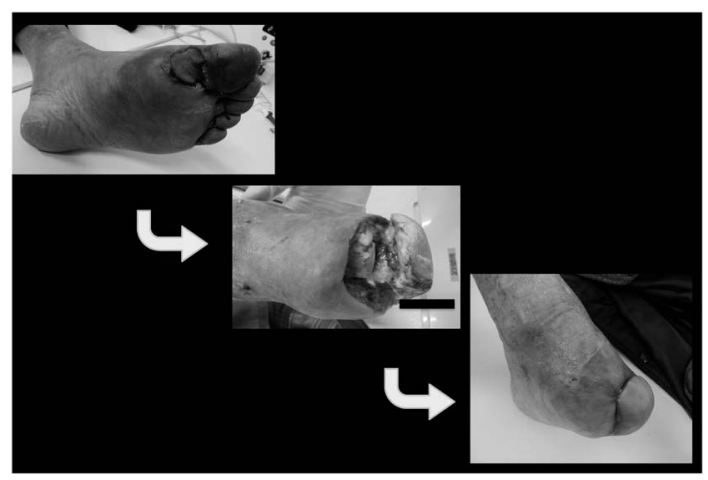

Fig. 2 The progress of leg wound after the distal bypass for critical limb ischemia.

After the bypass and Lisfranc amputation the wound did not heal and even the bone could see through the dehisced wound. But after the persistent treatment with vacuum assisted closure the wound finally healed and patient has visited outpatient clinic on foot for two years since then.

the patient has been visiting the hospital as an outpatient on foot for over two years. However, the patient did not follow a smooth therapeutic course after the healing of the wound. Endovascular treatment was performed on the superficial femoral artery stenosis proximal to the former bypass, and a distal bypass surgery was performed again on the contralateral limb that had developed CLI during the course. Ileus also occurred immediately after this surgery, which resulted in readmission to the hospital. The patient finally recovered via conservative therapy. As in this case, CLI patients must often undergo retreatment to the legs or repeated therapy for other disorders even after undergoing revascularization. Patient-orientated therapy that support patients until the end, could be achieved via multidisciplinary treatment.

\section{Multidisciplinary Treatment for Improving Prognosis}

PAD patients, especially those with CLI, have particularly poor prognosis in terms of survival, despite not having a poor prognosis of their limbs. ${ }^{8)}$ It is easy to focus on the treatment of the lower limbs, but the systemic condition must always be tracked to avoid the development of lifethreatening complications. For instance, in a 67-year-old male patient who was referred to our clinic with claudication after walking for $500 \mathrm{~m}$, conservative therapy was selected because the case was not that of CLI. Although there was no indication for invasive treatment in our hospital, we tried to screen other vascular diseases at his first examination. Double-vessel disease was detected via the abnormality in the electrocardiography and accordingly, percutaneous transluminal coronary angioplasty 


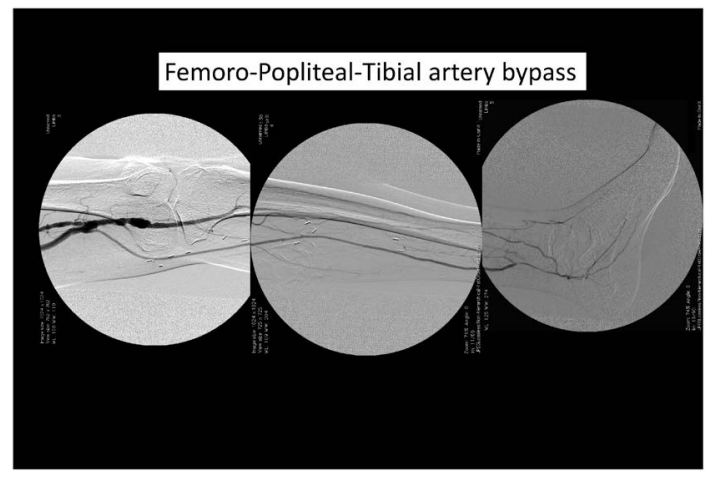

Fig. 3 Femoro-Popliteal-Tibial artery bypass performed in CLI patients 1 year after percutaneous coronary intervention and bilateral carotid artery stenting.

Table 1 Incidence of comorbidity in critical limb ischemia patients according to the National Clinical Database (NCD)/Japan Critical Limb Ischemia Database (JCLIMB) 2015

\begin{tabular}{lc}
\hline \multicolumn{1}{c}{ Critical limb ischemia patients } & 1,138 cases \\
\hline Diabetes mellitus & $67 \%$ \\
Hypertension & $73 \%$ \\
Dyslipidemia & $38 \%$ \\
Ischemic heart disease & $43 \%$ \\
Cerebral vascular disease & $22 \%$ \\
End-stage renal disease & $43 \%$ \\
History of malignancy & $8 \%$ \\
\hline
\end{tabular}

was performed in the department of cardiology. Moreover, high-grade stenosis was detected bilaterally in the internal carotid artery after careful analyses of carotid bruit; and bilateral carotid artery stenting was performed in the department of neurosurgery. Finally CLI developed in this patient during the follow-up, and femoral-poplitealposterior tibial artery bypass surgery was thus performed after a year in our department (Fig. 3). Two years later, the patient is well and continues to visit the hospital as an outpatient. As in this case, patients who finally end up with CLI often carry coronary artery diseases or cerebrovascular diseases that directly affect their prognosis; therefore, polyvascular disease must always be given strong consideration during treatment of such cases. ${ }^{9)}$ It is also necessary to build a system that allows close collaboration with specialists from other fields on a daily basis, so that professionals can consult each other causally whenever needed. The high incidence of complications of disorders in CLI patients is obvious from data from the Japan Critical Limb Ischemia Database's annual report ${ }^{10)}$ (Table 1); therefore, treatment, including screening tests, must be performed actively.

\section{Multidisciplinary Treatment for Preventing Increase in Severity}

We actively performed revascularization surgery that was mainly centered on bypass surgery on CLI patients every day, although the outcomes were not always favorable. In particular, several reports have shown poor prognosis in patients on hemodialysis or those with diabetes mellitus. Therefore, it is clear that prevention not to develop CLI is more important than to treat aggressively after progression to a severe state. However, it is extremely difficult to detect progression at an early stage, ${ }^{11)}$ and various data show that it is inappropriate to perform preventive endothelial solely based on imaging findings. We usually compare claudicants and CLI patients using parameters of endothelial functions and serum biomarkers to find the point of transition. ${ }^{12-15)}$ Nevertheless, so far, we have been unable to identify the appropriate parameters. Thus, we have developed a new system, the Takii Foot Scan for discovering inhospital patients with foot disorders at the early stages with the help of nurses. ${ }^{16)}$ At the time of hospitalization, the nurses in charge observe the feet of all patients and look for any foot disorders. This system runs with the great help of nurses. The doctor-in-charge normally makes careful observations in their specialized field but often focuses less on disorders of other fields that may be present in the patient. As a result, referral of patients with foot disorders may get delayed. Regarding this point, services of professional nurses who can check the patient overall without biases are required. This system functions more effectively because the report of the leg patients is done from ward nurse to gatekeeper nurse of the foot-care team without hesitation between the same occupations. With this approach, approximately $40 \%$ of the reported cases have benefited from the system. One patient whose CLI was discovered with the sake of this system treated successfully with the distal bypass and achieved limb salvage. This system has the potential to contribute to early discovery when it involves the use of the capabilities of medical staff, including nurses. Currently, we are constructing a new collaboration system based on this approach in the regional network. From the patients' perspective, it is understandable that they will feel discontent when a large hospital could not find their complicated disorder, even if it is not the main target lesions. Hospitals such as university hospitals usually offer enough specialized treatment, but early discovery of other disease should be attempted through multidisciplinary treatment and team approach by collaborating with medical staffs. ${ }^{17)}$

\section{Conclusion}

There is a range of therapeutic options for treating foot 


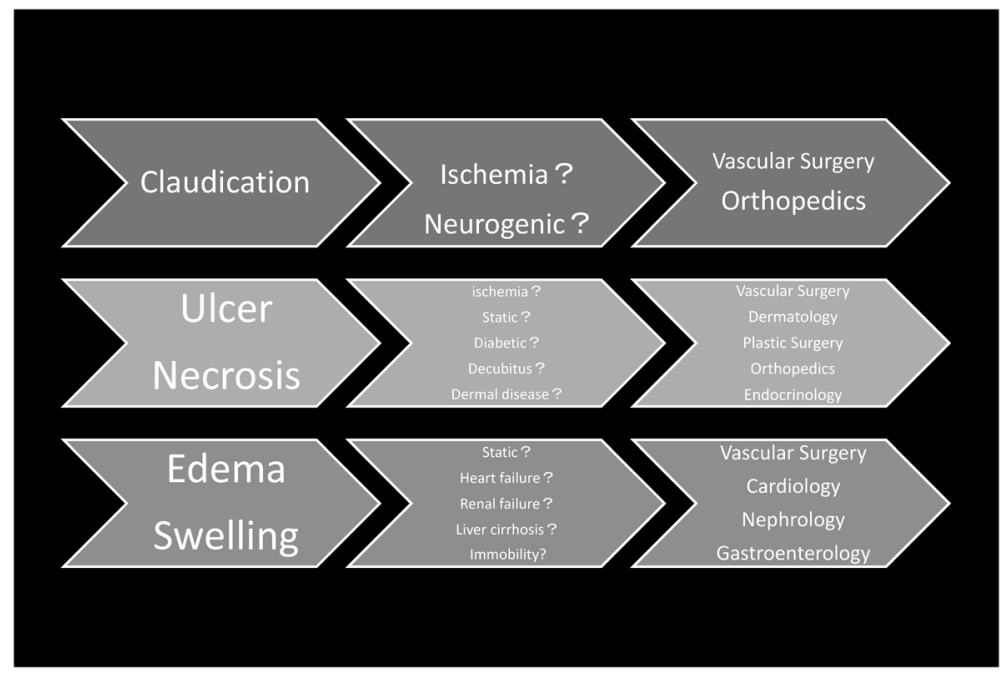

Fig. 4 Leg diseases are caused by various etiologies and treated in various specialists.

lesions depending on the causes and mechanisms of development (Fig. 4). It is clear that several different departments must collaborate to perform treatment on such patients. The treatment should involve not only physicians but medical staffs, because there are many aspects of treatment which can be covered only by medical staffs. Such capacity of medical staff members must be fully utilized. The current medical examinations performed on vascular disease and treatment of foot lesions are similar to the structure of a human pyramid. In such a case, each person contributes without visualizing the overall picture, but an excellent result is achieved. However, the pyramid will not be completed if any one of these people is missing. Vascular surgeons should become the person who takes the role of coordinator for completing this pyramid. I strongly emphasize that medical treatment and care performed for leg vascular disease should always be "total foot care," which indicates that the medical team should always perceive the body systemically, not only focusing on the leg or foot. The word "specialist" may imply the professional for very restricted field of disease, but true patient-orientated treatment cannot be achieved unless they possess the ability to closely cooperate with other physician and medical staff of multiple disciplines. Multidisciplinary treatment is therefore a key word for CLI treatment in the future.

\section{Disclosure Statement}

The author has no conflict of interest.

\section{Additional Note}

The main contents of this paper were presented in the 27th Educational Seminar of the Japanese Society for Vascular Surgery (May 11, 2018; Yamagata, Japan).

\section{References}

1) Norgren L, Hiatt WR, Dormandy JA, et al. Inter-Society Consensus for the Management of Peripheral Arterial Disease (TASC II). J Vasc Surg 2007; 45 Suppl S: S5-67.

2) Miyata $T$, Akazawa $H$, Akishita $M$, et al. Guidelines for the management of peripheral arterial occlusive diseases (JCS 2015) http://www.j-circ.or.jp/guideline/pdf/JCS2015_ miyata_h.pdf (Accessed: 10/20/2018). (in Japanese)

3) Akagi D, Hoshina K, Akai A, et al. Outcomes in patients with critical limb ischemia due to arteriosclerosis obliterans who did not undergo arterial reconstruction. Int Heart J 2018; 59: 1041-6.

4) Mills JL Sr, Conte MS, Armstrong DG, et al. The society for vascular surgery lower extremity threatened limb classification system: risk stratification based on Wound, Ischemia, and foot Infection (WIfI). J Vasc Surg 2014; 59: 220-34.el-2.

5) Aboyans V, Ricco JB, Bartelink MEL, et al. 2017 ESC guidelines on the diagnosis and treatment of peripheral arterial diseases, in collaboration with the European Society for Vascular Surgery (ESVS): document covering atherosclerotic disease of extracranial carotid and vertebral, mesenteric, renal, upper and lower extremity arteries endorsed by: the European Stroke Organization (ESO) The Task Force for the Diagnosis and Treatment of Peripheral Arterial Diseases of the European Society of Cardiology (ESC) and of the European Society for Vascular Surgery (ESVS). Eur Heart J 2018; 39: 763-816.

6) Ohki T. National survey of treatment for peripheral arterial disease in Japan reveals stent abuse and inappropriate treatment calling for enhanced professional autonomy. Journal of Japan Surgical Society 2016; 117: 404-8. (in Japanese)

7) Sumikawa M, Saitoh S, Kubota M. Questionnaire-based survey on foot care in patients with diabetes: a survey of the Japan Diabetes Society-certified educational and related educational facilities. Journal of the Japan Diabetes Society 2016; 59: 748-58. (in Japanese with English abstract)

8) van Haelst STW, Koopman C, den Ruijter HM, et al. Cardiovascular and all-cause mortality in patients with in- 
termittent claudication and critical limb ischaemia. Br J Surg 2018; 105: 252-61.

9) Yamazaki T, Goto S, Shigematsu H, et al. Prevalence, awareness and treatment of cardiovascular risk factors in patients at high risk of atherothrombosis in Japan. Circ J 2007; 71: 995-1003.

10) The Japanese Society for Vascular Surgery JCLIMB Committee, NCD JCLIMB Analytical Team. 2015 JAPAN Critical Limb Ischemia Database (JCLIMB) annual report. Ann Vasc Dis 2018; 11: 398-426.

11) Shirasu T, Hoshina K, Yamamoto S, et al. Poor prognosis in critical limb ischemia without pre-onset intermittent claudication. Circ J 2015; 79: 1618-23.

12) Komai H, Higami Y, Tanaka H, et al. Impaired flow-mediated endothelium-dependent and endothelium-independent vasodilation of the brachial artery in patients with atherosclerotic peripheral vascular disease. Angiology 2008; 59: 52-6.
13) Komai H, Shindo S, Sato M, et al. Reduced protein C activity might be associated with progression of peripheral arterial disease. Angiology 2015; 66: 584-7.

14) Komai H, Shibata R, Juri M, et al. Plasma adiponectin as a predictive factor of survival after a bypass operation for peripheral arterial disease. J Vasc Surg 2009; 50: 95-9.

15) Komai H, Miyama N, Sakashita H, et al. Plasma carnitine level in peripheral arterial disease. The Journal of Japanese College of Angiology 2016; 56: 103-8. (in Japanese with English abstract)

16) Sakasita H, Okubo M, Tanimura H, et al. Trial of early detection system for leg lesion by medical staffs, "Takii Footscan”. Japanese Journal of Foot Care 2017; 15: 65-8. (in Japanese)

17) Komai H. Treatment for peripheral arterial disease and team approach with medical staffs. Sogo Rihabiriteshon 2017; 45 : 801-6. (in Japanese) 